\title{
Mean-atom-trajectory model for the velocity autocorrelation function of monatomic liquids
}

\author{
Eric D. Chisolm, Brad E. Clements, and Duane C. Wallace \\ Theoretical Division \\ Los Alamos National Laboratory \\ Los Alamos, NM 87545
}

October 27, 2018

\begin{abstract}
We present a model for the motion of an average atom in a liquid or supercooled liquid state and apply it to calculations of the velocity autocorrelation function $Z(t)$ and diffusion coefficient $D$. The model trajectory consists of oscillations at a distribution of frequencies characteristic of the normal modes of a single potential valley, interspersed with position- and velocity-conserving transits to similar adjacent valleys. The resulting predictions for $Z(t)$ and $D$ agree remarkably well with MD simulations of $\mathrm{Na}$ at up to almost three times its melting temperature. Two independent processes in the model relax velocity autocorrelations: (a) dephasing due to the presence of many frequency components, which operates at all temperatures but which produces no diffusion, and (b) the transit process, which increases with increasing temperature and which produces diffusion. Because the model provides a single-atom trajectory in real space and time, including transits, it may be used to calculate all single-atom correlation functions.
\end{abstract}

\section{Introduction}

In order to explain the experimental fact that the specific heat of a solid changes little, while its self-diffusion coefficient changes greatly, when it melts 
to a liquid, Frenkel [1, 2] suggested that an atom in a liquid undergoes approximately harmonic vibrations about an equilibrium position, occasionally jumping from one equilibrium position to another; these jumps are responsible for self-diffusion. Using molecular dynamics calculations, Stillinger and Weber ([3]-[6]) demonstrated the presence of local many-particle minima ("inherent structures") in the potential surface underlying the liquid state, and they observed that "diffusion and fluid flow within a liquid may be interpreted as transitions between ... local minima" [5]. Building on these ideas, Zwanzig [7] studied the self-diffusion coefficient $D$, given in terms of the velocity autocorrelation function $Z(t)=\frac{1}{3}\langle\boldsymbol{v}(t) \cdot \boldsymbol{v}(0)\rangle$ by the Green-Kubo formula [8]

$$
D=\int_{0}^{\infty} Z(t) d t .
$$

For harmonic motion about a many-particle equilibrium position, $Z(t)$ is given by

$$
Z(t)=\frac{k T}{M} \int \rho(\omega) \cos (\omega t) d \omega
$$

where $\rho(\omega)$ is the density of normal mode frequencies. (The derivation of this formula is discussed in [7] and performed in Section II of [31].) Zwanzig suggested that jumps between equilibrium positions will have the effect of multiplying this expression by a factor $\exp \left(-t / \tau_{\mathrm{zw}}\right)$, where the "hopping time" $\tau_{\mathrm{zw}}$ is characteristic of the time between jumps. Much effort has been devoted to developing these ideas into full harmonic theories of liquid dynamics, particularly theories of self-diffusion in liquids and supercooled liquids ([9]- 64]). In most theories one finds $\rho(\omega)$ by expanding the potential energy to second order around each of some set of configurations, diagonalizing the second-order term in the potential (often called the dynamical matrix) to find the frequency distribution for that configuration, and averaging over all configurations chosen. This is done in one of two different ways. In quenched normal mode (QNM) theories [9, 40, 41, 43, 60, 62], $\rho(\omega)$ is calculated at several potential minima and averaged, while in instantaneous normal mode (INM) theories ([10]- [37], [46]- 51], [63], [64]) $\rho(\omega)$ is averaged over a thermal distribution of configurations, with no special emphasis placed upon configurations in potential valleys. This difference manifests itself in the fact that in INM theories, the configuration-averaged $\rho(\omega)$ usually includes both real and imaginary frequencies (corresponding to stable and unstable normal modes), since most configurations will not lie at the bottoms of valleys, while in QNM theories only real frequencies are represented. In addition, the QNM den- 
sity of states for a given system will in general be temperature-independent, while the INM density will depend strongly on temperature. The two most prominent ways to determine $\tau_{\mathrm{zw}}$ are (a) to extract it from the imaginary frequency INM distribution, developed most notably by Keyes (10-25), and (b) to set $\tau_{\mathrm{zw}}^{-1}$ equal to the long-time decay rate of the "cage correlation function" of Rabani, Gezelter, and Berne [60, 62]. (Another theory uses Cao and Voth's frequency-dependent multiplicative factor [44, 45].) Notice that none of these theories attempt to model the actual motion of a diffusing particle in the liquid and then calculate $Z(t)$ from this motion; they try to model the effects of diffusion on the autocorrelation function directly.

The theory of $Z(t)$ we propose differs from those discussed above both in how we determine $\rho(\omega)$ and how we model the effects of diffusion. In Section 2, we suggest a density of states of the QNM type that also incorporates insights drawn from studies of the potential energy surface of liquid $\mathrm{Na}$ by Clements and Wallace [55, 56]. These studies were undertaken to test a theory of monatomic liquid dynamics proposed by Wallace [52] that has been applied previously with some success to the thermodynamics of a wide variety of liquid metals [53] and an earlier study of $Z(t)$ [54], and the present work is also intended to lend credence to that theory. We propose a model for the process of diffusion based on the following observations. The system moves in a set of nearly harmonic many-particle valleys, and the motion within each valley may be analyzed into normal modes of vibration about the valley minimum. The motion of the system from one valley to another is called a transit, and it corresponds to a change in the equilibrium positions of a small group of atoms. When a transit occurs involving a given atom, or one of its neighbors, the normal mode eigenvector components for that atom will change, and since this can happen many times during a single vibrational period (as we will see in Section 3), the eigenvectors will not necessarily provide a useful basis for describing the motion. This suggests that an independent atom model will be a good theoretical starting point [54]. (Notice that this argument does not apply to INM, where the eigenvectors change continuously with the motion, instead of discontinuously only at transits.) Therefore, we propose a mean-atom-trajectory model which describes a single average particle in the diffusing liquid actually transiting between single-particle equilibrium positions, and from this model we calculate $Z(t)$ directly. In Section 3 we compare our predictions with molecular dynamics (MD) simulations of liquid $\mathrm{Na}$ over a very broad range of temperatures, and in Section 4 we discuss our results. 


\section{The model}

\subsection{Density of states}

Wallace [52 has predicted that in any monatomic liquid the many-body potential valleys can be divided into three categories: the few crystalline valleys; so-called "symmetric" valleys which retain some remnant of the crystal system's symmetry; and "random" valleys, in which no crystal symmetries remain. He argued further that the random valleys should greatly outnumber the symmetric ones (thus controlling the statistical mechanics of the liquid), and that all random valleys should have the same distribution of normal mode frequencies. Wallace and Clements [55, 56] have verified these predictions for liquid $\mathrm{Na}$, as well as discovering criteria that one can use to determine whether the system is in a symmetric or random valley when nondiffusing. From Fig. 7 of [55] one can construct a distribution $\rho(\omega)$ for liquid $\mathrm{Na}$ that will be valid whenever the system remains in a random valley; this $\rho(\omega)$ is shown in Fig. 1. However, since we actually have the set of normal mode frequencies $\left\{\omega_{\lambda}\right\}$ found in [55], we can use them in Eq. (2) directly, so our nondiffusing $Z(t)$ is

$$
Z(t)=\frac{1}{3 N-3} \frac{k T}{M} \sum_{\lambda} \cos \left(\omega_{\lambda} t\right),
$$

where $N$ is the number of particles in the system, and the number of normal modes is $3 N-3$ because the three zero-frequency modes corresponding to center of mass motion are not excited. This is our model $Z(t)$ when the system remains in a single random valley without diffusion. Note that because all of the random valleys have the same $\rho(\omega)$, an average over quenched configurations is unnecessary.

\subsection{Motion of an Average Particle}

Our first goal is to construct a model for the motion of an average particle that reproduces Eq. (3) for $Z(t)$ in the absence of transits. Since the system is transiting with overwhelming likelihood from random valley to random valley, and since all the random valleys have the same frequency distribution, it is sensible to model an average particle's motion in terms of oscillations at those frequencies, or

$$
\boldsymbol{r}(t)=\boldsymbol{R}+\boldsymbol{u}(t)
$$




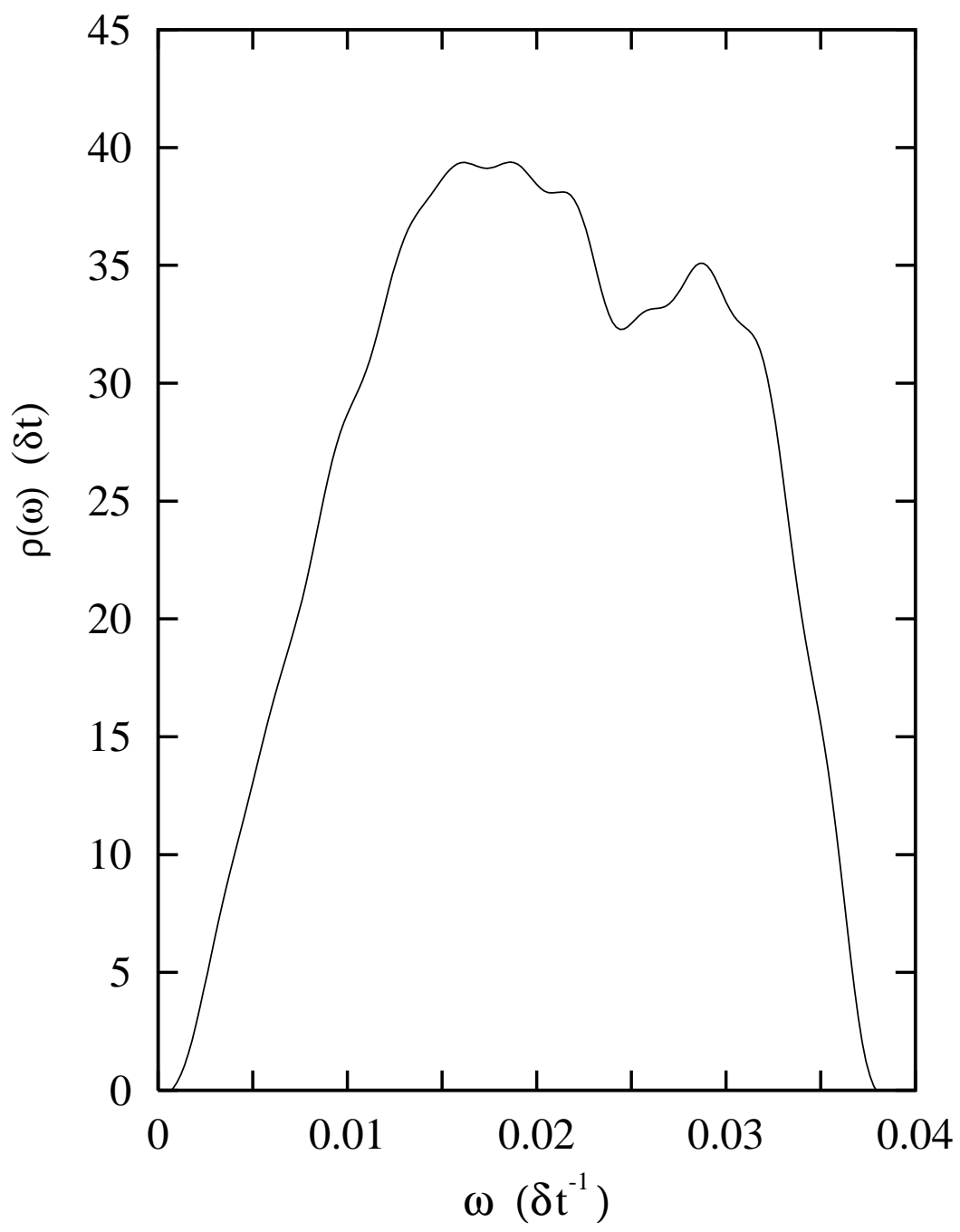

Figure 1: $\rho(\omega)$ for liquid Na constructed from the set of frequencies in Fig. 7 of [5.5]. Here $\delta t$ is the MD timestep $\left(1.4 \times 10^{-15} \mathrm{~s}\right)$. 


$$
=\boldsymbol{R}+\sum_{\lambda} \boldsymbol{w}_{\lambda} \sin \left(\omega_{\lambda} t+\alpha_{\lambda}\right),
$$

where the particle's position $\boldsymbol{r}(t)$ is divided into a center of oscillation $\boldsymbol{R}$ and oscillations $\boldsymbol{u}(t)$ about that center, and the parameters in $\boldsymbol{u}(t)$ aside from the $\omega_{\lambda}$ have yet to be determined. Let us assume that the values of the phases $\alpha_{\lambda}$ are randomly distributed among the particles; then one calculates $Z(t)$ from Eq. (4) by differentiating to find $\boldsymbol{v}(t)$, computing the product $\boldsymbol{v}(t) \cdot \boldsymbol{v}(0)$, and averaging over each of the $\alpha_{\lambda}$ separately; the result is

$$
Z(t)=\frac{1}{6} \sum_{\lambda}\left|\boldsymbol{w}_{\lambda}\right|^{2} \omega_{\lambda}^{2} \cos \left(\omega_{\lambda} t\right) .
$$

Eq. (5) becomes Eq. (3) with the choice

$$
\boldsymbol{w}_{\lambda}=\sqrt{\frac{1}{N-1} \frac{2 k T}{M \omega_{\lambda}^{2}}} \hat{\boldsymbol{w}}_{\lambda}
$$

where $\hat{\boldsymbol{w}}_{\lambda}$ is an arbitrarily chosen unit vector. Thus Eq. (4) with the phases $\alpha_{\lambda}$ randomly chosen and $\boldsymbol{w}_{\lambda}$ given by Eq. (6), with the unit vectors $\hat{\boldsymbol{w}}_{\lambda}$ also randomly chosen, constitute our mean-atom-trajectory model when the system is not diffusing.

To include diffusion in our model, we must incorporate both the rate at which transits occur and their effect on the particle's motion. We will allow the rate $\nu$ to be a temperature-dependent parameter that we will determine in Section 3 by fitting to MD simulations (so the probability of a transit in small time $\Delta t$ is $\nu \Delta t$ ), and we assume that the transit occurs instantaneously (the particle simply crosses the surface separating distinct valleys), so it must conserve both the particle's position $\boldsymbol{r}(t)$ and velocity $\boldsymbol{v}(t)$. To be more specific, we assume that the transit occurs in the forward direction, so that the center of the new valley lies an equal distance away from the particle but on the opposite side from the center of the old valley. Let $\boldsymbol{r}^{\text {before }}(t)$, $\boldsymbol{R}^{\text {before }}$, and $\boldsymbol{u}^{\text {before }}(t)$ be the position parameters from Eq. (4) before the transit, and let $\boldsymbol{r}^{\text {after }}(t), \boldsymbol{R}^{\text {after }}$, and $\boldsymbol{u}^{\text {after }}(t)$ be the parameters after; then our assumption of a forward transit implies that $\boldsymbol{u}^{\text {after }}(t)=-\boldsymbol{u}^{\text {before }}(t)$, and this together with $\boldsymbol{r}^{\text {before }}(t)=\boldsymbol{r}^{\text {after }}(t)$ implies

$$
\boldsymbol{R}^{\text {after }}=\boldsymbol{R}^{\text {before }}+2 \boldsymbol{u}^{\text {before }}(t) \text {. }
$$

This is the change in $\boldsymbol{R}$ produced by a transit. We choose to leave the unit vectors $\hat{\boldsymbol{w}}_{\lambda}$ in Eq. (6) unaffected by transits, leaving only the effect 
on the phases $\alpha_{\lambda}$ to be determined. They must change in such a way as to reverse the sign of $\boldsymbol{u}(t)$ but conserve $\boldsymbol{v}(t)$; since $\boldsymbol{u}(t)$ is a sum of sines while $\boldsymbol{v}(t)$ is a sum of cosines, this is easily done by reversing the signs of the arguments $\left(\omega_{\lambda} t+\alpha_{\lambda}\right)$ in Eq. (4). Let the transit occur at time $t_{0}$; then $\omega_{\lambda} t_{0}+\alpha_{\lambda}^{\text {after }}=-\left(\omega_{\lambda} t_{0}+\alpha_{\lambda}^{\text {before }}\right)$ so

$$
\alpha_{\lambda}^{\text {after }}=-2 \omega_{\lambda} t_{0}-\alpha_{\lambda}^{\text {before }}
$$

Thus, a transit is implemented at time $t_{0}$ by leaving the $\hat{\boldsymbol{w}}_{\lambda}$ alone and making the substitutions

$$
\begin{gathered}
\boldsymbol{R} \rightarrow \boldsymbol{R}+2 \boldsymbol{u}\left(t_{0}\right) \\
\alpha_{\lambda} \rightarrow-2 \omega_{\lambda} t_{0}-\alpha_{\lambda} .
\end{gathered}
$$

This conserves $\boldsymbol{r}(t)$, reverses the sign of $\boldsymbol{u}(t)$, and conserves $\boldsymbol{v}(t)$.

Now the model consists of two parts. (a) Between transits, the average particle moves nondiffusively as given by Eq. (4) and (6), with the phases $\alpha_{\lambda}$ and unit vectors $\hat{\boldsymbol{w}}_{\lambda}$ assigned randomly. (b) In each small time interval $\Delta t$ a transit occurs with probability $\nu \Delta t$; if it occurs, it replaces $\boldsymbol{R}$ and the $\alpha_{\lambda}$ with new values according to Eq. (9). With the addition of transits, we can no longer express $\boldsymbol{r}(t)$ and $\boldsymbol{v}(t)$ in closed form at all times, so we no longer have a closed form for $Z(t)$; but the model can be implemented easily on a computer, and then the data from the run can be used to calculate $Z(t)$ in a manner analogous to an MD simulation. We turn to comparison of the predictions of this model with MD results next.

\section{Comparison with MD}

The MD setup used to test our model is that described in [55] with two changes: $N=500$ in all runs and the MD timestep was reduced to $\delta t=0.2 t^{*}$, where $t^{*}=7.00 \times 10^{-15} \mathrm{~s}$ is the natural timescale defined in [55]. (The system's mean vibrational period $\tau=2 \pi / \omega_{\text {rms }}$, where $\omega_{\text {rms }}$ is the rms average of the normal mode frequency distribution, is approximately $287 \delta t$.) We performed equilibrium runs of the system at $6.69 \mathrm{~K}, 22.3 \mathrm{~K}, 216.3 \mathrm{~K}, 309.7$ $\mathrm{K}, 425.0 \mathrm{~K}, 664.7 \mathrm{~K}$, and $1022.0 \mathrm{~K}$; at the lower two temperatures the system is not diffusing (as can be seen from the system's mean square displacement, or from the integral of $Z(t)$ ), and the system is diffusing otherwise. Since $T_{m}=371.0 \mathrm{~K}$ for $\mathrm{Na}$ at this density, our simulations range from the glassy 
regime to nearly three times the melting temperature. We then ran the model for various values of $\nu$, adjusting until the model matched the value of the first minimum of $Z(t)$ at each temperature. The values of $\nu$ that we fit for all temperatures are given below; Figs. 2 through 7 compare the model's predictions with the MD results for $\hat{Z}(t)=Z(t) / Z(0)$. The model requires $\nu=0$ for both nondiffusing states, so they are presented together in Fig. 2 .

\begin{tabular}{rl}
$T(\mathrm{~K})$ & $\nu\left(\tau^{-1}\right)$ \\
\hline 6.69 & 0.0 \\
22.3 & 0.0 \\
216.3 & 0.35018 \\
309.7 & 0.60276 \\
425.0 & 0.83985 \\
664.7 & 1.24858 \\
1022.0 & 1.68774
\end{tabular}

Notice that in all diffusing cases $\nu$ is of the same order of magnitude as $\tau^{-1}$, indicating roughly one transit per mean vibrational period, as predicted in 57 and noted in Section 1.

The most obvious trend in $\hat{Z}(t)$ is that its first minimum is rising with increasing $T$; this is the primary reason for the increasing diffusion coefficient $D$. Note that the model is able to reproduce this most important feature quite satisfactorily. In fact, all fits of the model to the MD results capture their essential features, but we do see systematic trends in the discrepancies. First, note that the location of the first minimum barely changes at all in the model as $\nu$ is raised, but in MD the first minimum moves steadily to earlier times as the temperature rises. The first minimum occurs at a time roughly equal to half of the mean vibrational period (recall $\tau=287 \delta t$ ), so the steady drift backward suggests that the MD system is sampling a higher range of frequencies at higher $T$. Also, for the three lowest diffusing temperatures the model tends to overshoot the MD result in the vicinity of the first two maxima after the origin, and at the highest two temperatures this overshoot is accompanied by a positive tail that is slightly higher than the (still somewhat long) tail predicted by MD. These overshoots should clearly affect the diffusion coefficient $D$. To check this, we calculated the reduced diffusion coefficient $\hat{D}$, the integral of $\hat{Z}(t)$, which is related to $D$ by $D=(k T / M) \hat{D}$. The results are compared to the values of $\hat{D}$ calculated from the MD runs in Fig. 8. In all of the diffusing cases, the model overestimates $\hat{D}$ by roughly the same amount, which we take to be the effect of the overshoots 


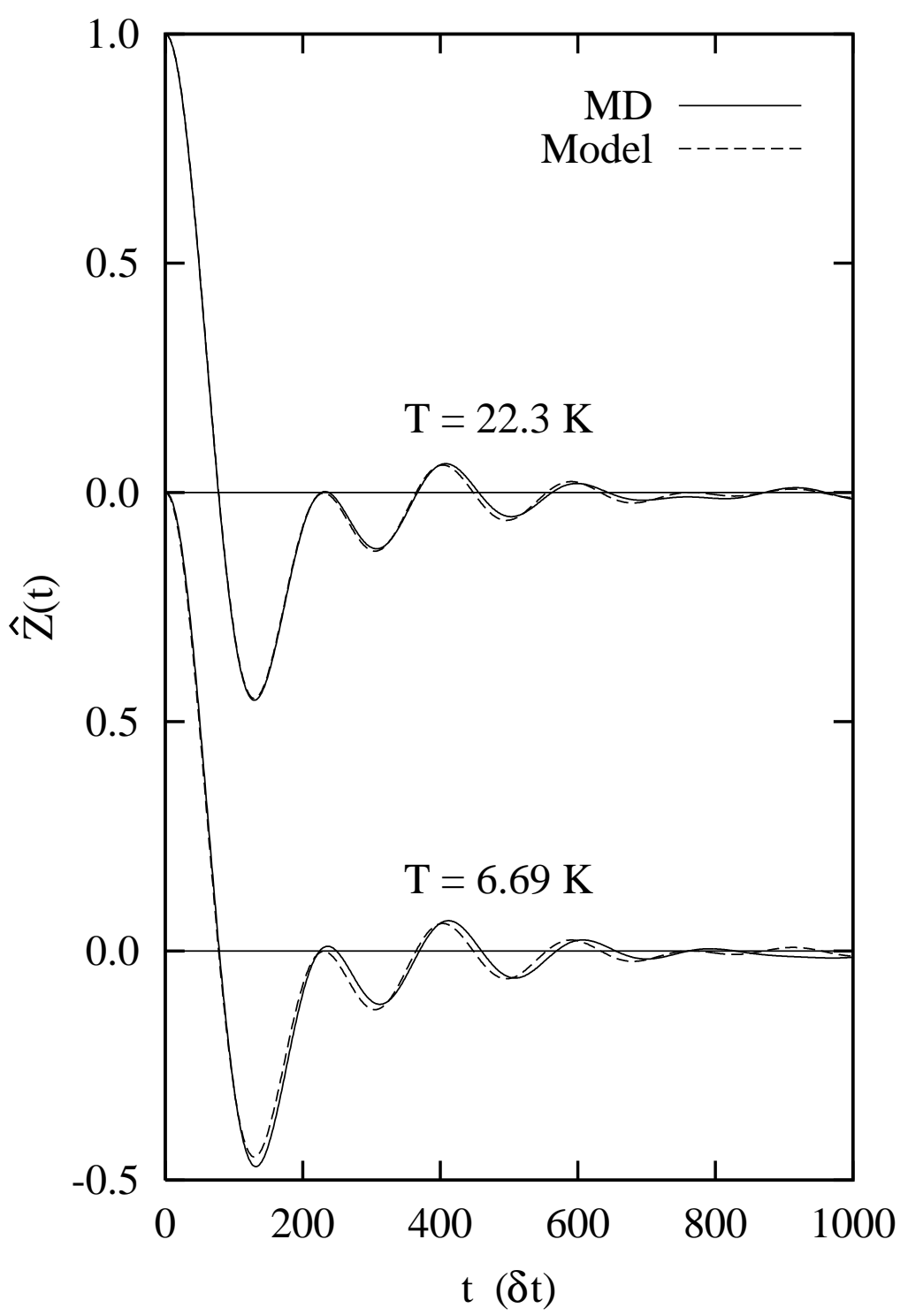

Figure 2: The model prediction for $\hat{Z}(t)$ at $\nu=0.0$ compared with the MD results for glassy liquid $\mathrm{Na}$ at $T=6.69 \mathrm{~K}$ and $T=22.3 \mathrm{~K}$. 


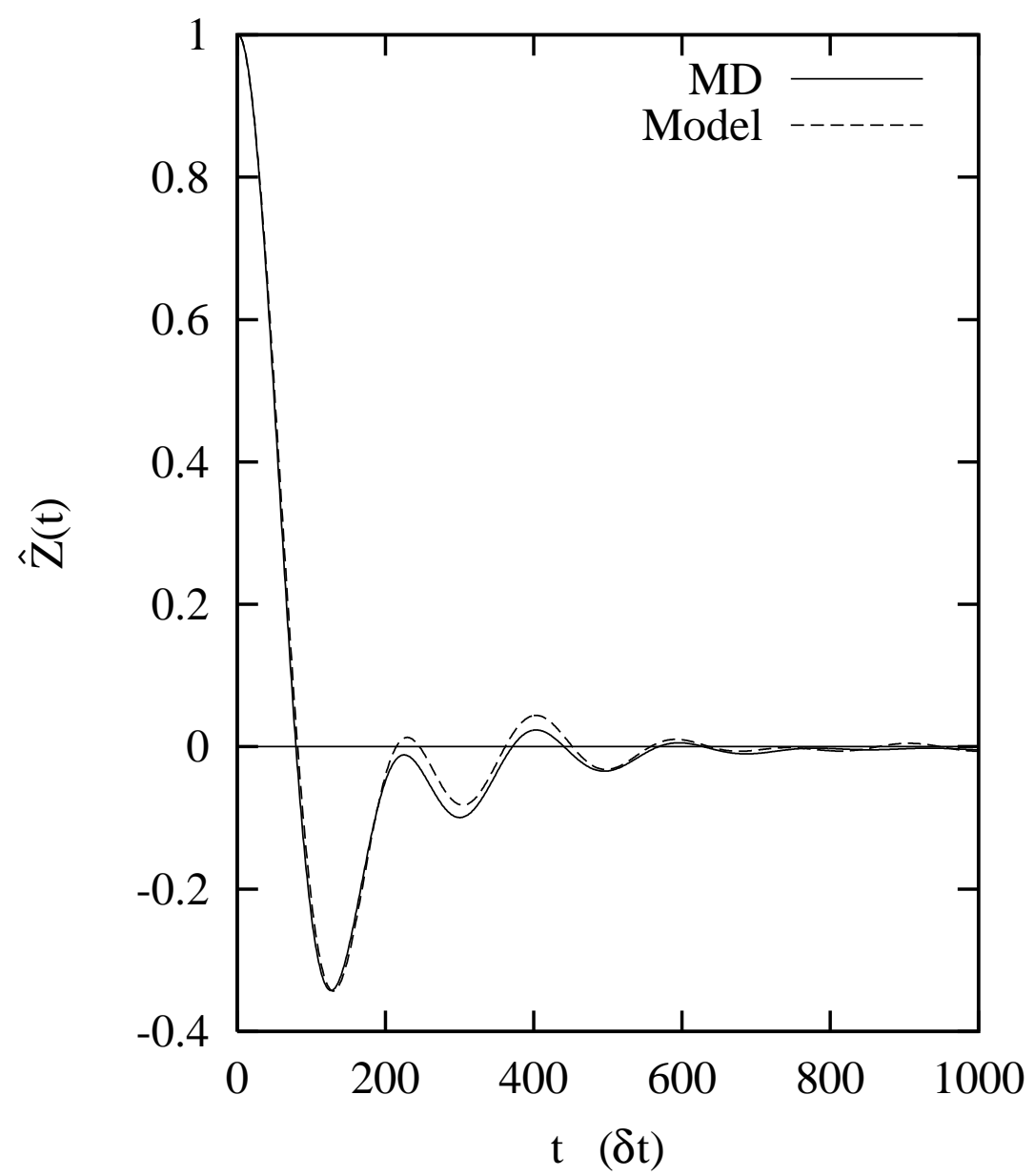

Figure 3: The model prediction for $\hat{Z}(t)$ at $\nu=0.35018 \tau^{-1}$ compared with the MD result for supercooled liquid $\mathrm{Na}$ at $T=216.3 \mathrm{~K}$. 


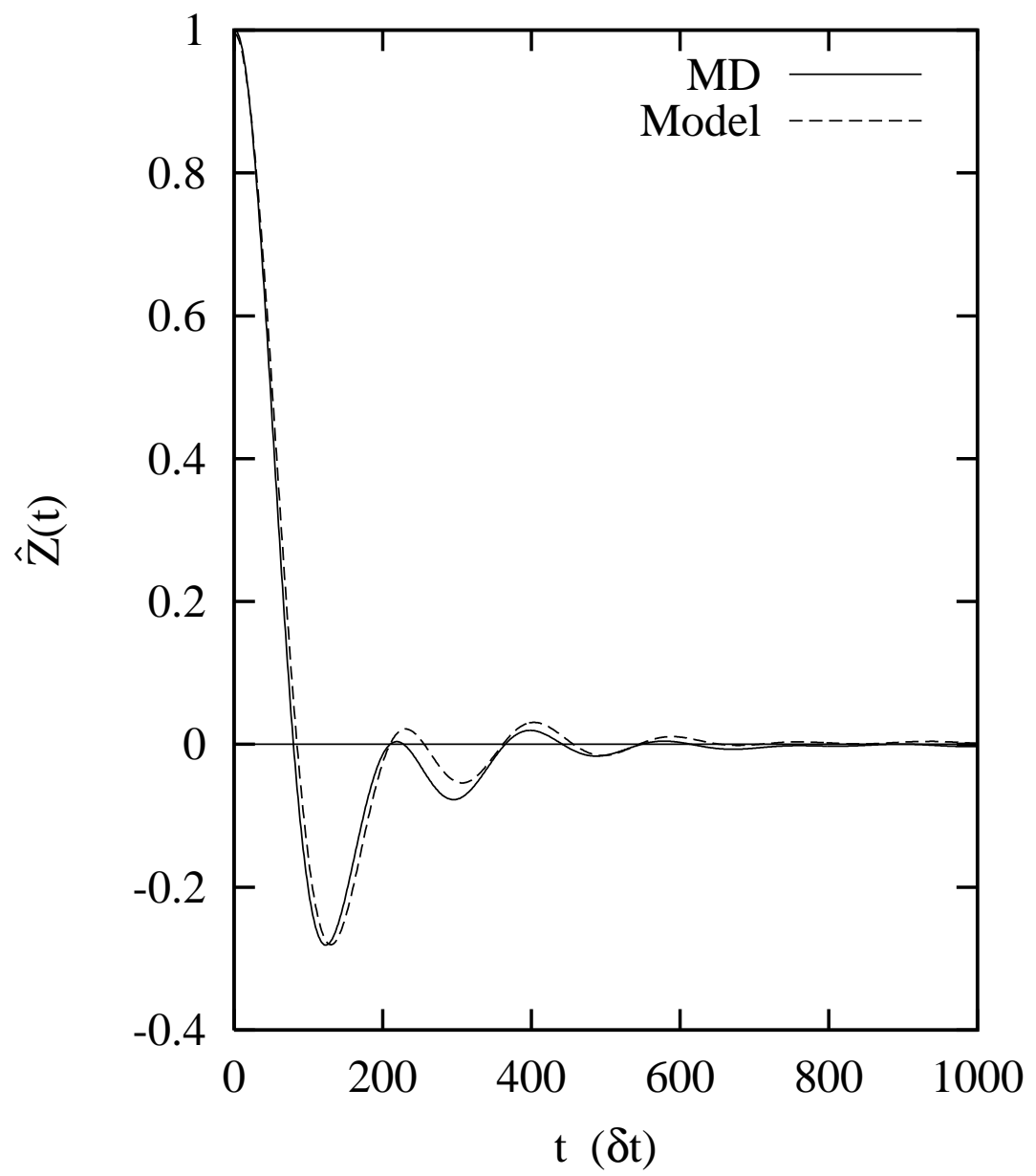

Figure 4: The model prediction for $\hat{Z}(t)$ at $\nu=0.60276 \tau^{-1}$ compared with the MD result for supercooled liquid $\mathrm{Na}$ at $T=309.7 \mathrm{~K}$. 


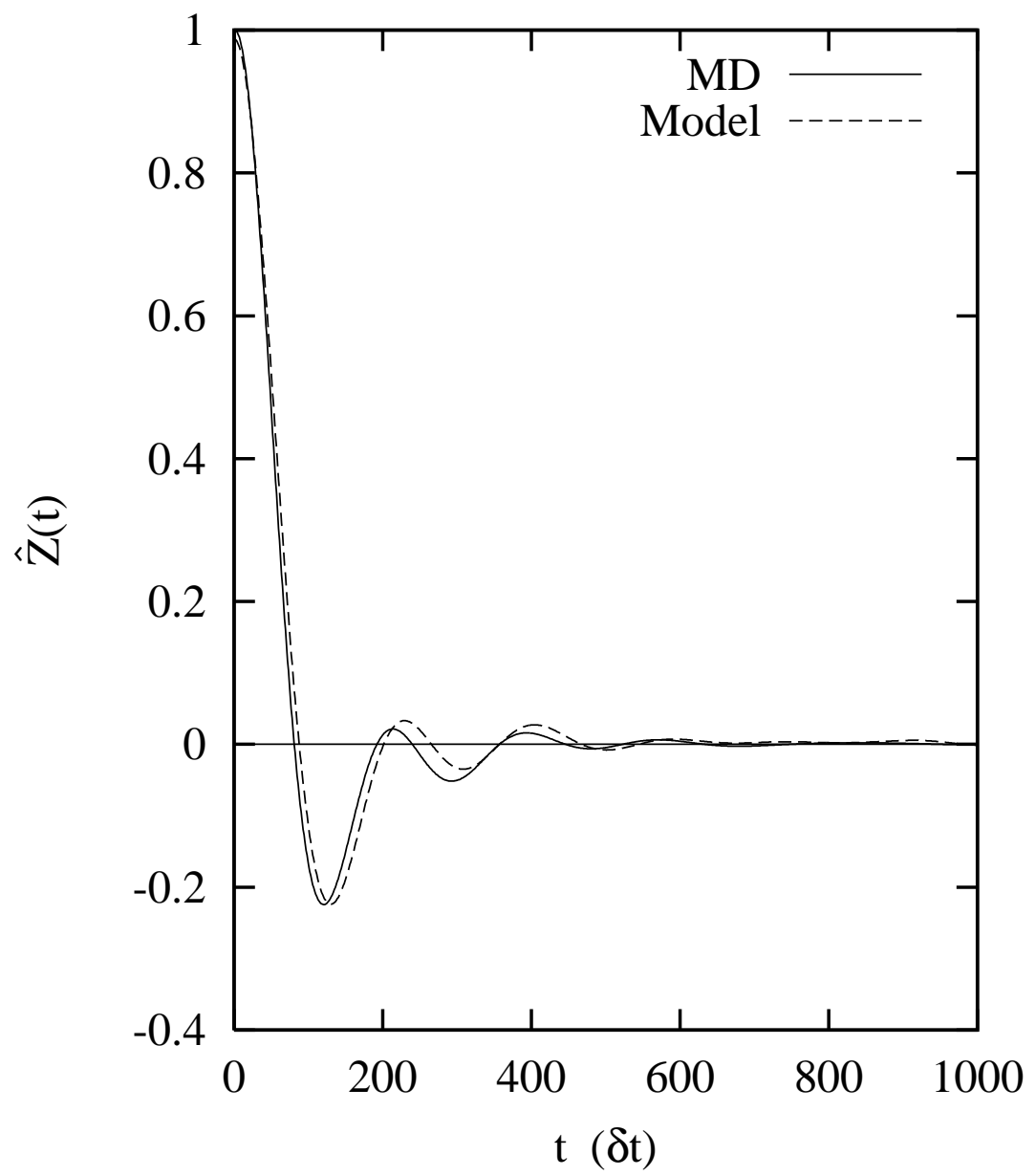

Figure 5: The model prediction for $\hat{Z}(t)$ at $\nu=0.83985 \tau^{-1}$ compared with the MD result for liquid $\mathrm{Na}$ at $T=425.0 \mathrm{~K}$. 


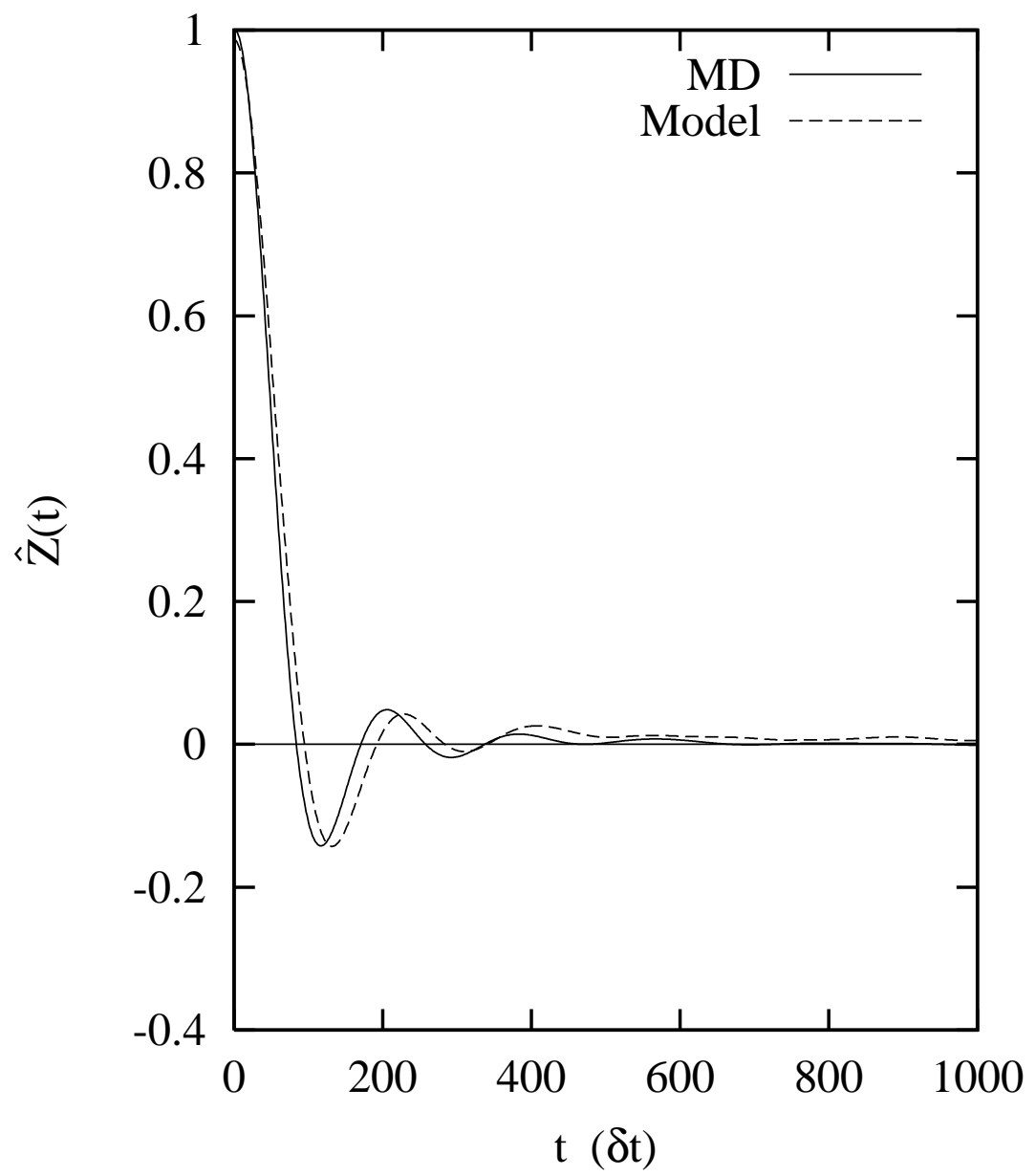

Figure 6: The model prediction for $\hat{Z}(t)$ at $\nu=1.24858 \tau^{-1}$ compared with the MD result for liquid $\mathrm{Na}$ at $T=664.7 \mathrm{~K}$. 


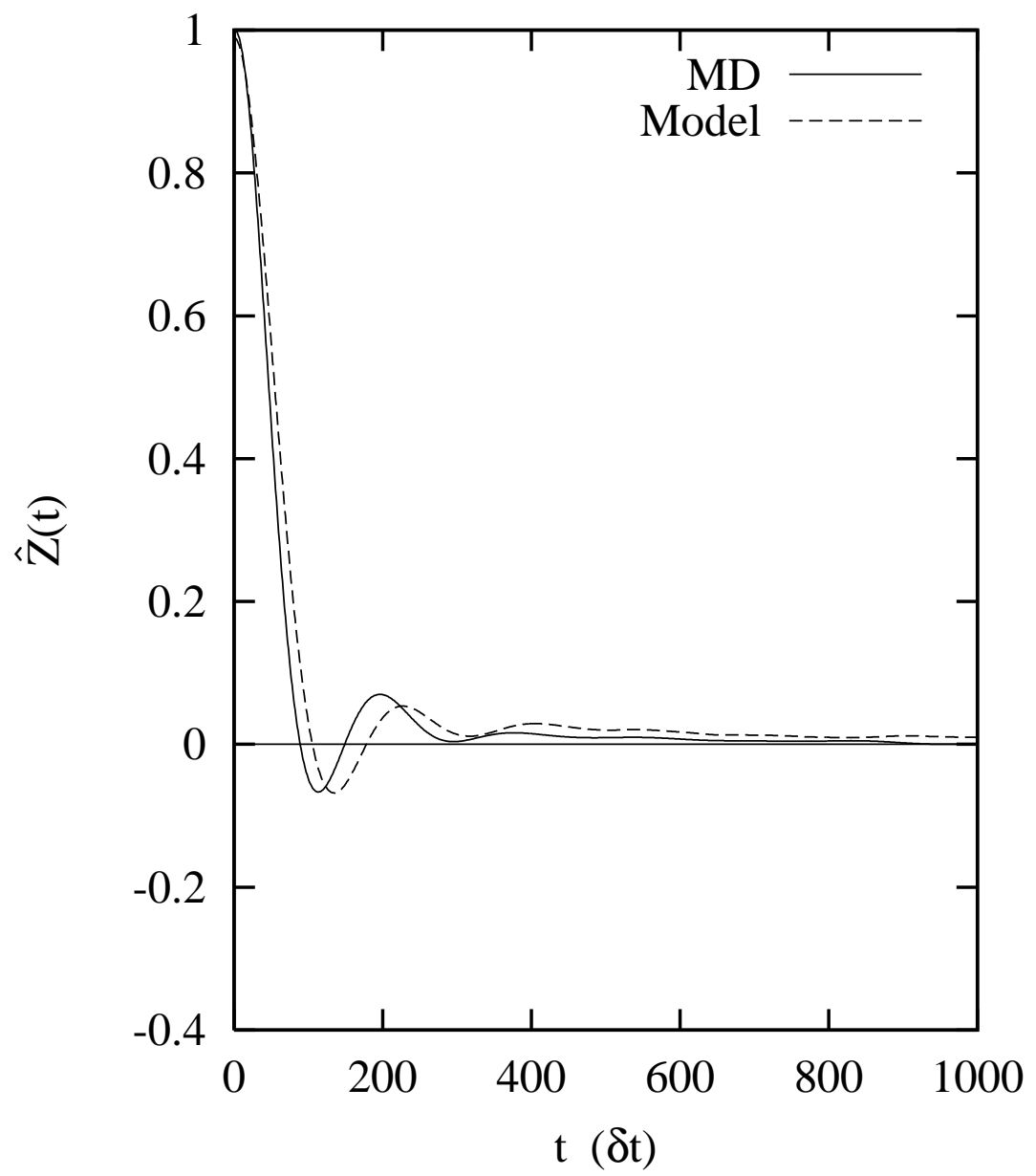

Figure 7: The model prediction for $\hat{Z}(t)$ at $\nu=1.68774 \tau^{-1}$ compared with the MD result for liquid $\mathrm{Na}$ at $T=1022.0 \mathrm{~K}$. 


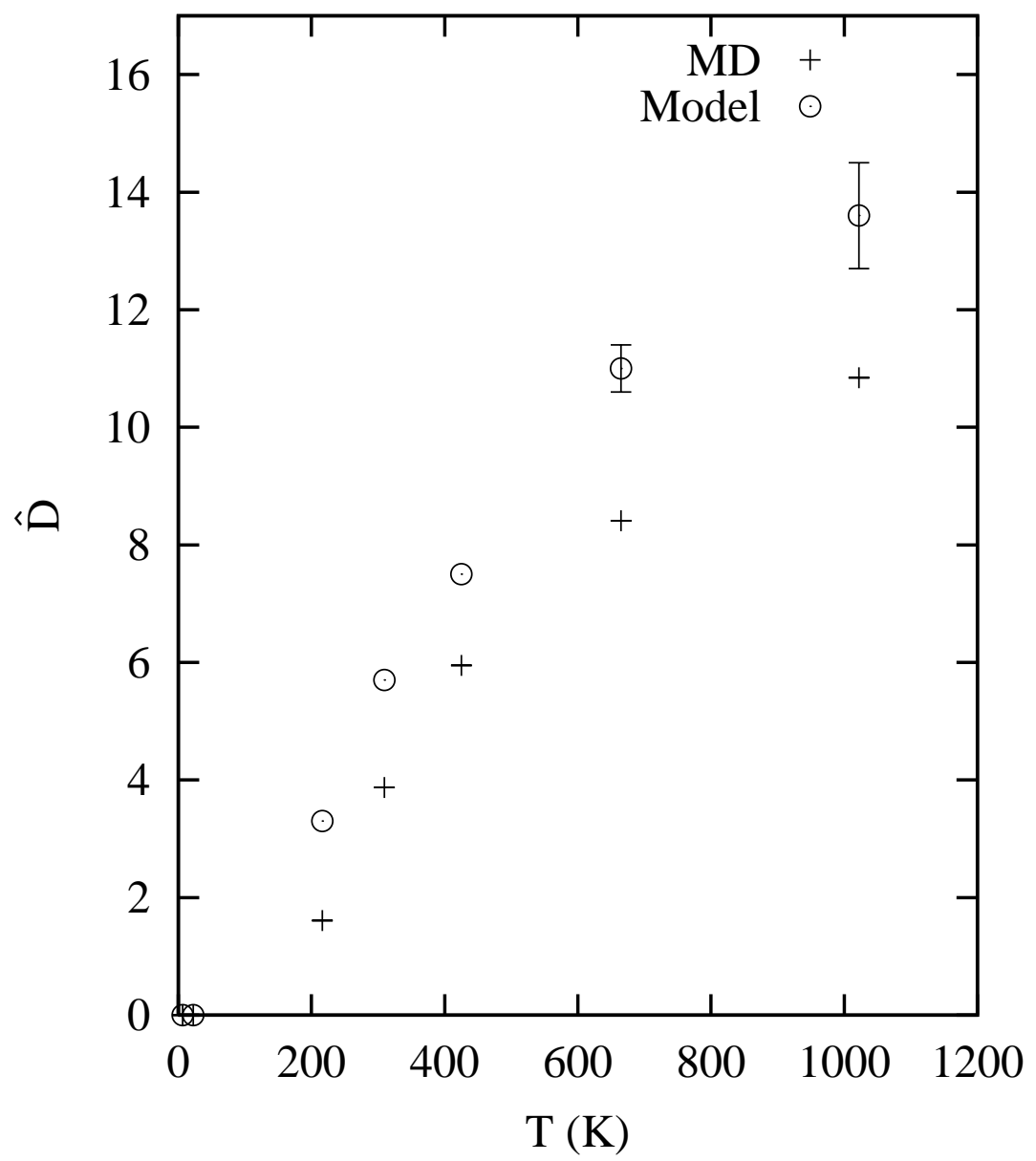

Figure 8: $\hat{D}$ as a function of $T$ for both the model and MD. 
at the first two maxima. At the higher temperatures the discrepancy is also higher, presumably due to the model's long tail.

\section{Conclusions}

We have presented a single-atom model of a monatomic liquid that provides a unified account of diffusing and nondiffusing behavior. The nondiffusing motion is modeled as a sum of oscillations at the normal mode frequencies (Eq. (4) and (6) ); self-diffusion is accounted for in terms of instantaneous transits between wells, which occur at a temperature-dependent rate $\nu$. Since this model gives a simple and straightforward account of the motion itself, it can easily be used to calculate any single-atom correlation function one wishes; here we have focussed on $Z(t)$ and its integral $D$. The relaxation of correlations expressed by the decay of $Z(t)$ arises here from two distinct processes: Dephasing as a result of the large number of frequencies in the single-valley motion, and transits between valleys. The dephasing effect produces relaxation but not diffusion: It causes $Z(t)$ to decay but its integral remains zero. On the other hand, transits certainly contribute to relaxation, but in addition they raise the first minimum of $Z(t)$ substantially, increasing its integral and providing a nonzero $D$.

Most other workers in the field have studied Lennard-Jones or molecular liquids; the only other work with liquid $\mathrm{Na}$ we have found is $\mathrm{Wu}$ and Tsay's INM analysis [48, 49], with which our results are of comparable quality. This is remarkable in light of the fact that our model of the transit process is exceedingly simple; one would expect that a more realistic model would produce even better results. We are also pleased to see that the model retains its validity from the glassy regime to well beyond the liquid's melting temperature.

In light of these results, answers to the following questions are worth pursuing. Do other monatomic liquids exhibit the same division of their potential valleys into random and symmetric that liquid $\mathrm{Na}$ does? (It is known that LJ Ar does [58.) How harmonic are these valleys? How similar are their frequency distributions? Fig. 2 shows that in $\mathrm{Na}$ the valleys are very nearly perfectly harmonic, and we expect the same qualitative potential surface for all nearly-free-electron metals (a total of 24 elemental liquid metals), but other liquids might show more pronounced anharmonicities, and those would need to be accounted for in the model. Could a more sophisticated 
model of the transit process (as opposed to simply transiting forward) produce the shift in the first minimum and smaller long-time tail shown in MD? Can one develop a theory to predict the transit probability $\nu$ ? (Such a theory would be conceptually related to the decay of the cage correlation function of [60, 62].) Finally, how can these ideas be applied to theories of other transport coefficients, such as bulk and shear viscosities? Future work will focus on answering these questions.

\section{Acknowledgements}

This work was supported by the U. S. DOE through contract W-7405-ENG36 .

\section{References}

[1] J. Frenkel, Z. Phys. 35, 652 (1926).

[2] J. Frenkel, Kinetic Theory of Liquids (Clarendon, Oxford, 1946), Chap. III, Sec. 1.

[3] F. H. Stillinger and T. A. Weber, Phys. Rev. A 25, 978 (1982).

[4] F. H. Stillinger and T. A. Weber, Phys. Rev. A 28, 2408 (1983).

[5] T. A. Weber and F. H. Stillinger, J. Chem. Phys. 80, 2742 (1984).

[6] F. H. Stillinger and T. A. Weber, J. Chem. Phys. 83, 4767 (1985).

[7] R. Zwanzig, J. Chem. Phys. 79, 4507 (1983).

[8] J. P. Hansen and I. R. McDonald, Theory of Simple Liquids (Academic, London, 1986), Ch. 7, pp. 199-202.

[9] A. Pohorille, L. R. Pratt, R. A. LaViolette, M. A. Wilson, and R. D. McElroy, J. Chem. Phys. 87, 6070 (1987).

[10] G. Seeley and T. Keyes, J. Chem. Phys. 91, 5581 (1989).

[11] B. Madan, T. Keyes, and G. Seeley, J. Chem. Phys. 92, 7565 (1990).

[12] B. Madan, T. Keyes, and G. Seeley, J. Chem. Phys. 94, 6762 (1991). 
[13] G. Seeley, T. Keyes, and B. Madan, J. Chem. Phys. 95, 3847 (1991).

[14] B. Madan and T. Keyes, J. Chem. Phys. 98, 3342 (1993).

[15] P. Moore and T. Keyes, J. Chem. Phys. 100, 6709 (1994).

[16] T. Keyes, J. Chem. Phys. 101, 5081 (1994).

[17] T. Keyes, J. Chem. Phys. 103, 9810 (1996).

[18] T. Keyes, G. V. Vijayadamodar, and U. Zurcher, J. Chem. Phys. 106, 4651 (1997).

[19] W.-X. Li and T. Keyes, J. Chem. Phys. 107, 7275 (1997).

[20] T. Keyes, J. Phys. Chem. A 101, 2921 (1997).

[21] U. Zurcher and T. Keyes, Phys. Rev. E 55, 6917 (1997).

[22] W.-X. Li, T. Keyes, and F. Sciortino, J. Chem. Phys. 108, 252 (1998).

[23] T. Keyes, W.-X. Li, and U. Zurcher, J. Chem. Phys. 109, 4693 (1998).

[24] W.-X. Li and T. Keyes, J. Chem. Phys. 111, 5503 (1999).

[25] T. Keyes and J. T. Fourkas, J. Chem. Phys. 112, 287 (2000).

[26] B.-C. Xu and R. M. Stratt, J. Chem. Phys. 91, 5613 (1989).

[27] B.-C. Xu and R. M. Stratt, J. Chem. Phys. 92, 1923 (1990).

[28] K. Ganguly and R. M. Stratt, J. Chem. Phys. 97, 1980 (1992).

[29] Z. Chen and R. M. Stratt, J. Chem. Phys. 97, 5687 (1992).

[30] Z. Chen and R. M. Stratt, J. Chem. Phys. 97, 5696 (1992).

[31] M. Buchner, B. M. Ladanyi, and R. M. Stratt, J. Chem. Phys. 97, 8522 (1992).

[32] Y. Wan and R. M. Stratt, J. Chem. Phys. 100, 5123 (1994).

[33] R. M. Stratt and M. Cho, J. Chem. Phys. 100, 6700 (1994). 
[34] M. Cho, G. R. Fleming, S. Saito, I. Ohmine, and R. M. Stratt, J. Chem. Phys. 100, 6672 (1994).

[35] R. M. Stratt, Acc. Chem. Res. 28, 201 (1995).

[36] R. M. Stratt, Int. J. Thermophys. 18, 899 (1997).

[37] E. F. David and R. M. Stratt, J. Chem. Phys. 109, 1375 (1998).

[38] T.-M. Wu and R. F. Loring, J. Chem. Phys. 97, 8568 (1992).

[39] T.-M. Wu and R. F. Loring, J. Chem. Phys. 99, 8936 (1993).

[40] I. Ohmine and H. Tanaka, Chem. Rev. 932545 (1993).

[41] S. Saito and I. Ohmine, J. Chem. Phys. 108, 240 (1998).

[42] R. Vallauri and F. J. Bermejo, Phys. Rev. E 51, 2654 (1995).

[43] G. V. Vijayadamodar and A. Nitzan, J. Chem. Phys. 103, 2169 (1995).

[44] J. Cao and G. A. Voth, J. Chem. Phys. 102, 3337 (1995).

[45] J. Cao and G. A. Voth, J. Chem. Phys. 103, 4211 (1995).

[46] S. D. Bembenek and B. B. Laird, Phys. Rev. Lett. 74, 936 (1995).

[47] S. D. Bembenek and B. B. Laird, J. Chem. Phys. 104, 5199 (1995).

[48] T.-M. Wu and S.-F. Tsay, J. Chem. Phys. 105, 9281 (1996)

[49] T.-M. Wu and S.-F. Tsay, Phys. Rev. B 58, 27 (1998).

[50] T.-M. Wu, W.-J. Ma, and S.-F. Tsay, Physica A 254, 257 (1998).

[51] T.-M. Wu, W.-J. Ma, and S. L. Chang, J. Chem. Phys. 113, 274 (2000).

[52] D. C. Wallace, Phys. Rev. E 56, 4179 (1997).

[53] D. C. Wallace, Phys. Rev. E 57, 1717 (1998).

[54] D. C. Wallace, Phys. Rev. E 58, 538 (1998).

[55] D. C. Wallace and B. E. Clements, Phys. Rev. E 59, 2942 (1999). 
[56] B. E. Clements and D. C. Wallace, Phys. Rev. E 59, 2955 (1999).

[57] D. C. Wallace, Phys. Rev. E 60, 7049 (1999).

[58] E. D. Chisolm, B. E. Clements, and D. C. Wallace, unpublished.

[59] J. D. Gezelter, E. Rabani, and B. J. Berne, J. Chem. Phys. 107, 4618 (1997).

[60] E. Rabani, J. D. Gezelter, and B. J. Berne, J. Chem. Phys. 107, 6867 (1997).

[61] J. D. Gezelter, E. Rabani, and B. J. Berne, J. Chem. Phys. 109, 4695 (1998).

[62] E. Rabani, J. D. Gezelter, and B. J. Berne, J. Chem. Phys. 110, 3444 (1999).

[63] F. Sciortino and P. Tartaglia, Phys. Rev. Lett. 78, 2385 (1997).

[64] N. Krämer, M. Buchner, and Th. Dorfmüller, J. Chem. Phys. 109, 1912 (1998). 